\title{
Influence sur l'environnement de la mise au gabarit européen de navigation de la vallée de l'Aisne
}

PAR

\section{Cocozza}

Service spécial de la Navigation Belgique-Paris-Est

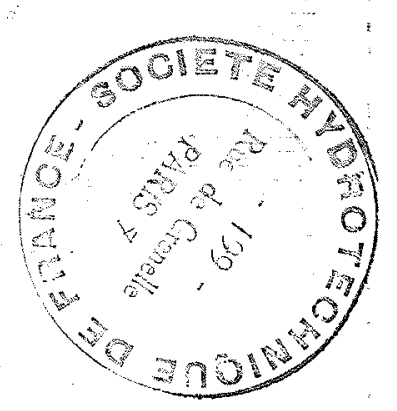

\section{Quelques rappels sur le réseau français}

Un simple coup d'œil jeté, même par un profane, sur une carte des voies navigables françaises, montre à l'évidence le caractère incomplet de la situation actuelle (fig. 1).

En effet, cinq grandes voies sont au gabarit international (de 3200 à $5000 \mathrm{t}$ ); ce sont la Seine, la liaison DunkerqueValenciennes, la Moselle, le Rhin et le Rhône à l'aval de Lyon. Ces voies ne sont reliées entre elles que par des voies au gabarit Freycinet de 250 à 400 t; le matériel moderne ne peut donc passer de l'une à l'autre et étouffe sur chacun de ces tronçons.

D'où l'idée déjà ancienne de relier ces voies par de grandes liaisons inter-bassins, au nombre de trois : la liaison Rhin-Rhône, la liaison Seine-Nord (reliant le bassin de la Seine aux voies du Nord de la France) et la liaison Seine-Est reliant le bassin de la Seine à l'Est de la France et en particulier à la Moselle.

\section{La liaison Seine-Est}

La liaison Seine-Est reliera la Seine à la Moselle en passant par Conflans-Sainte-Honorine, Creil, Compiègne, Soissons, Reims, Châlons-sur-Marne, Sainte-Menehould, Ambly et Toul.

Un premier tronçon, dont l'aménagement avait été retenu par le Ve Plan puis par le VI ${ }^{e}$, sera terminé fin avril 1975.
Il emprunte la vallée de l'Oise depuis son confluent avec la Seine jusqu'à Compiègne.

Un second tronçon avait été retenu pour le VI ${ }^{\ominus}$ Plan. Ce tronçon reliera Compiègne à Reims en empruntant la vallée de l'Aisne sur $90 \mathrm{~km}$, puis un petit affluent (la Loivre) sur $25 \mathrm{~km}$ environ.

A la différence du premier tronçon «Conflans-Compiègne » dont l'aménagement n'a nécessité que la construction de nouvelles écluses et quelques rescindements, la réalisation du second tronçon "Compiègne-Reims ». implique un remodelage complet, aussi bien du tracé que du profil en long de la voie.

Si le projet n'avait pas été étudié très soigneusement, on pouvait craindre un bouleversement complet des paysages et des équilibres écologiques sur plusieurs dizaines de kilomètres de vallée. Le service de navigation de Compiègne a donc pris l'initiative de confier (printemps 1973) au Centre d'Etudes Techniques de l'Equipement de Lille une étude sur l'environnement du canal à grand gabarit CompiègneSoissons-Reims.

Cette étude a consisté essentiellement à réaliser une photographie très précise de la situation actuelle, à mettre au point une méthode d'analyse des incidences sur l'environnement du projet et à proposer des solutions pour résoudre des problèmes déjà connus ou qui sont apparus au cours de l'étude, c'est-à-dire essentiellement:

- la traversée du massif forestier Compiègne-Laigue;

- la conservation des méandres de l'Aisne dans la partie amont de la vallée;

- les difficultés créées par le dépôt des 30 millions de $\mathrm{m}^{3}$ de déblais qui seront extraits tout au long de $115 \mathrm{~km}$ de voie;

- les problèmes ponctuels de traitement paysager des écluses, des ponts, des traversées d'agglomération, etc. 


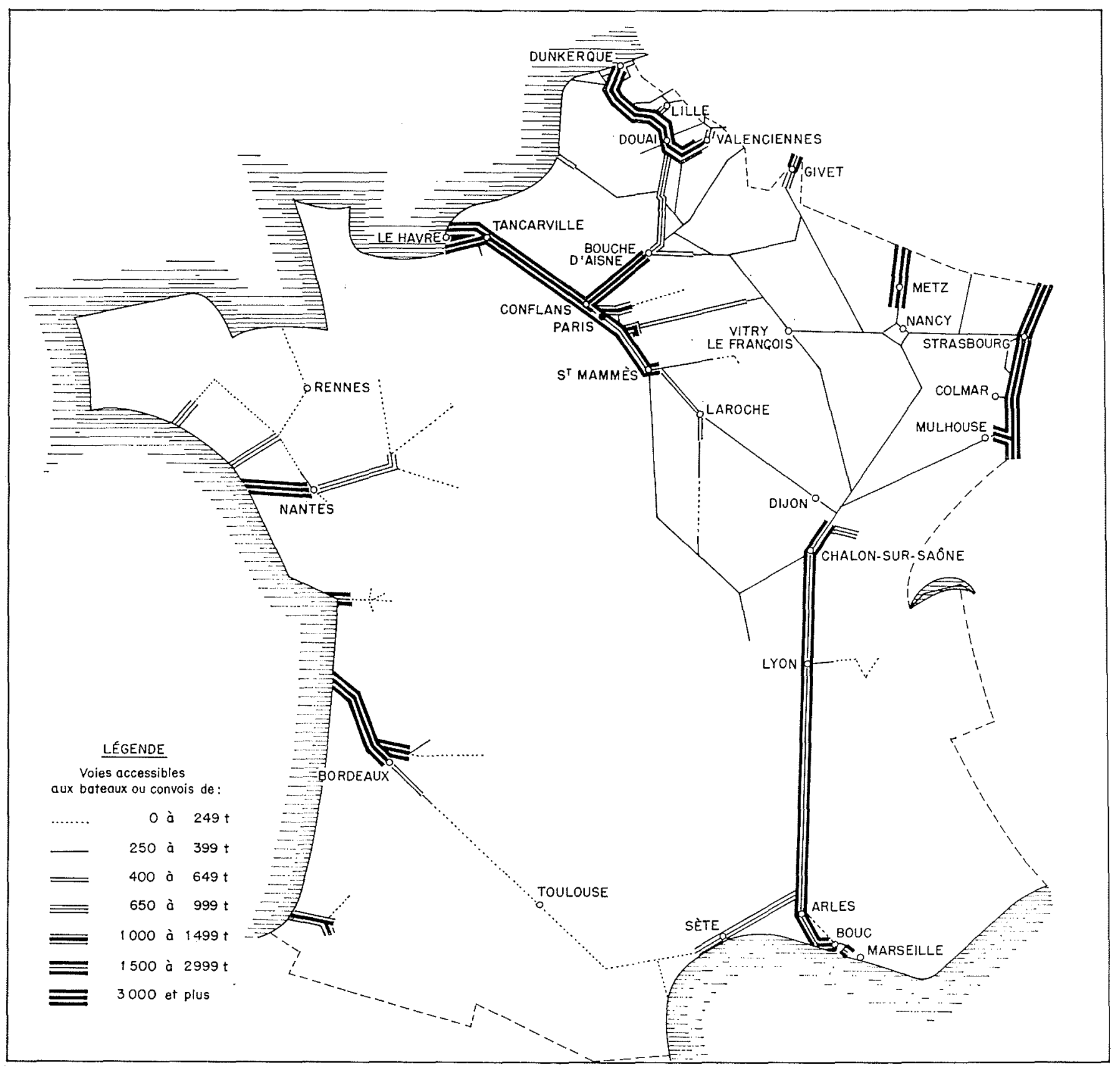

$1 /$

\section{La situation actuelle}

De Compiègnes à Reims, la voie navigable actuelle, à petit gabarit, emprunte successivement l'Aisne canalisée depuis Compiègne jusqu'à Soissons, le canal latéral à l'Aisne, de Soissons à Berry-au-Bac et, enfin, le canal de l'Aisne à la Marne, de Berry-au-Bac à Reims.

La vue perspective de la vallée de l'Aisne (fig. 2) prise dans l'axe de la vallée, dans la direction d'ouest en est, montre au premier plan l'important massif forestier de Compiègnes-Laigue-Ourscamps, au second plan la rivière enserrée entre les rebords boisés des plateaux du Soissonnais et du Laonnois et, enfin, à l'arrière-plan, l'horizon ample de la plaine Champenoise.

Les paysages qui se succèdent d'aval en amont sont d'une extrême diversité.
Paysage de la continuité de l'ensemble forestier de Compiègne-Laigue-Ourscamps, forêts de plaine s'étendant sur plus de 20000 ha d'un seul tenant, à peine interrompues par la coupure introduite par le cours de la rivière (fig. 3).

Dans l'espace réduit que constituent les rares interstices agricoles qui subsistent entre la forêt de Compiègne et celle de Laigue, se sont implantés des villages pittoresques dont la silhouette s'intègre parfaitement au site.

Mais immédiatement à l'orée des massifs, l'industrie exerce sa pression et dénature le site (fig. 4).

Ailleurs, les lisières forestières sont rognées par le développement des résidences secondaires, bloquant les échanges d'un massif à l'autre.

Il en est de même au niveau des interstices agricoles situés entre les deux massifs, menacés d'empiètement par l'extension de l'urbanisation. 


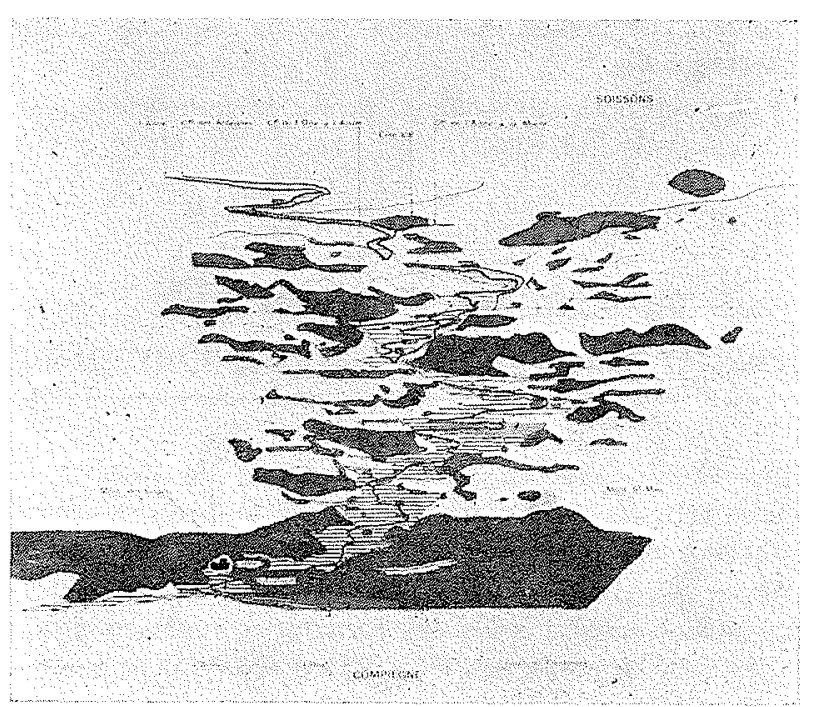

2/

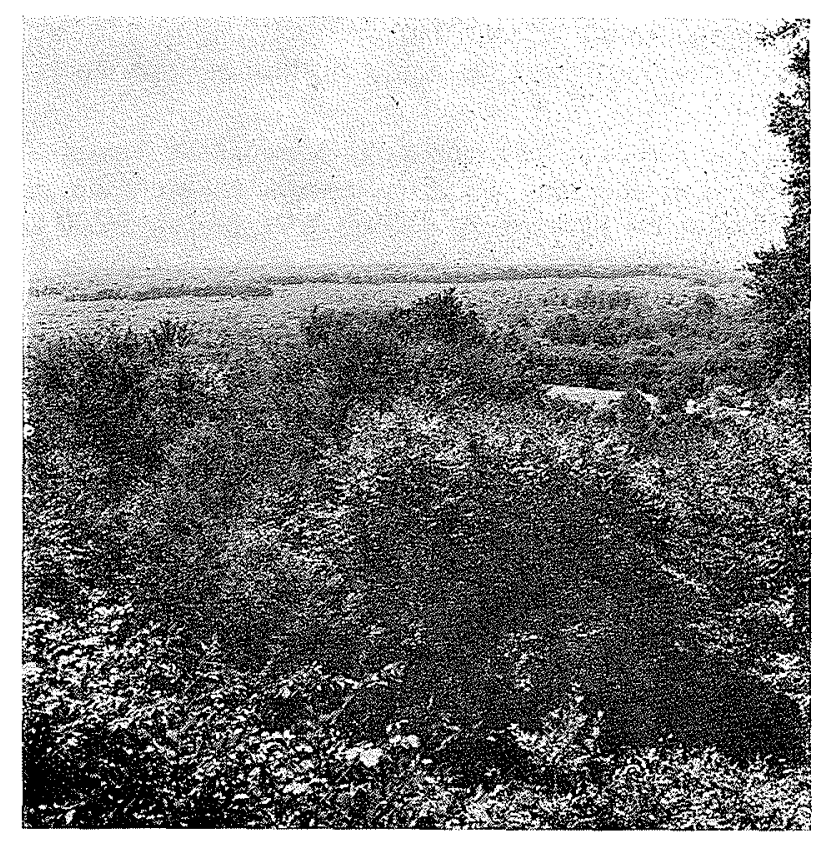

3/

Au-delà de l'ensemble forestier, s'étend jusqu'à Soissons un secteur de vallée enserré entre les cuestas boisés dominant l'Aisne, marqué par la présence de nombreux bosquets et parcelles de peupleraies parsemant la plaine alluviale et agricole (fig. 5).

L'agglomération de Soissons occupe un vaste élargissement de la vallée.

A l'est de Soissons, l'occupation humaine s'estompe. Le paysage est dominé par l'Aisne, rivière aux nombreux méandres bordés d'une végétation riche et variée (fig. 6).

A partir de Berry-au-Bac, on quitte le secteur de la vallée de l'Aisne proprement dite.

Le paysage est celui de la plaine découverte de la Champagne. Le tracé du futur canal s'infléchit vers le sud et emprunte la vallée de la Loivre jusqu'à Reims.

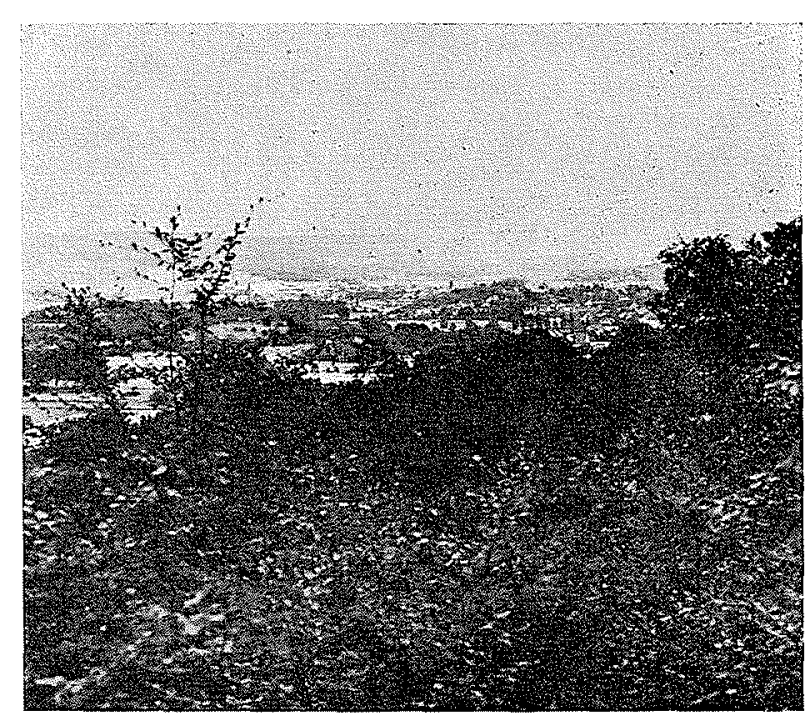

$4 /$

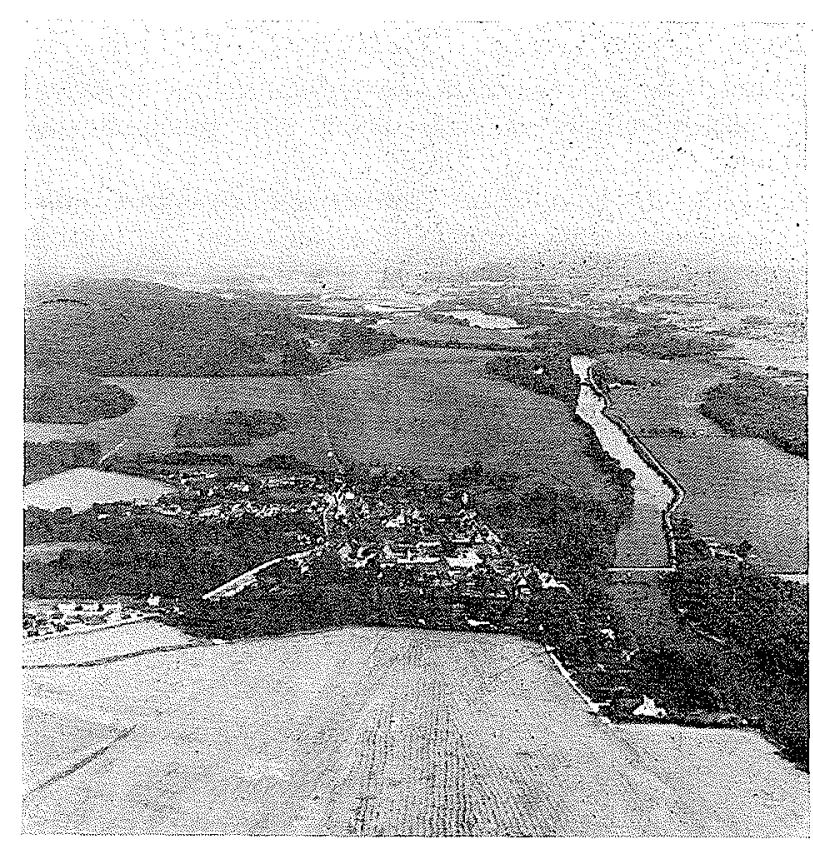

5/

\section{L'analyse des sites}

Cette reconnaissance sommaire de l'écologie et des paysages a été affinée par un inventaire détaillé, par photo-interprétation des éléments de l'occupation du sol.

Analyse de la végétation, de l'habitat, recensement des zones de gravières en exploitation ou en voie de colonisation par la végétation, recensement des zones industrielles, etc. (fig. 7) ont abouti à une délimitation de sites élémentaires caractérisés par la dominance ou la diversité des éléments écologiques qu'ils rassemblent et par leur ordonnancement dans l'espace en espaces ouverts, semi-ouverts ou fermés (fig. 8).

L'intérêt de ces sites a été défini suivant les critères de rareté, de vulnérabilité tant du point de vue écologique que 


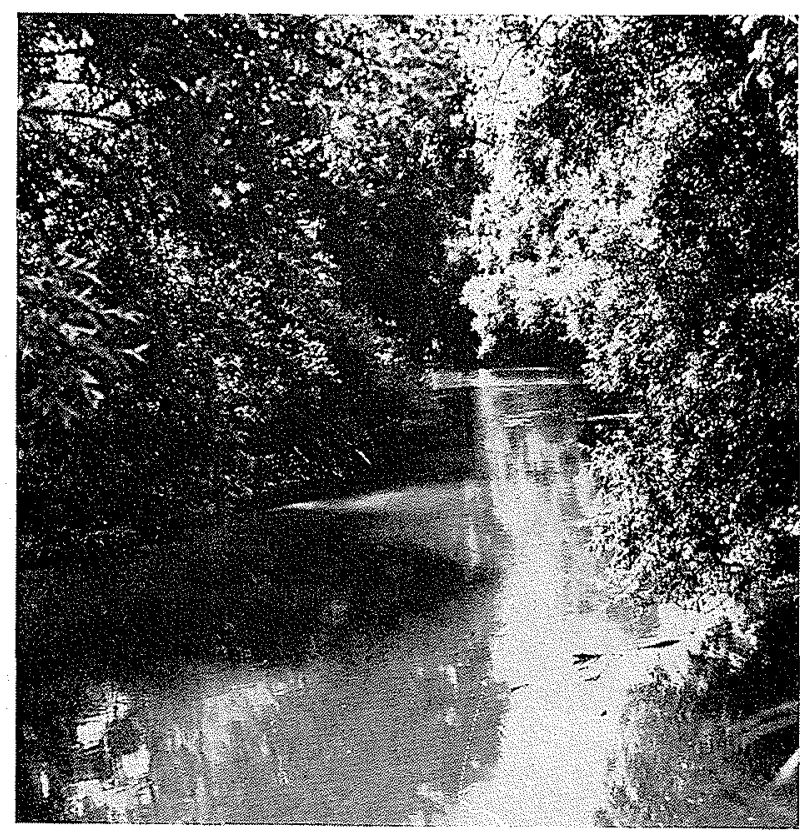

6/

paysager. Ont été également cartographiés les principaux sites archéologiques détectés grâce aux reconnaissances aériennes.

La prise en compte de l'intérêt des différents sites a abouti à un schéma simplifié d'organisation de la vallée par secteur, affecté chacun d'une vocation spécifique.

1) Secteur de la valiée protégée entre Choisy-au-Bac et Berneuil;

2) Secteur de vallée industrielle entre Berneuil et Vic-surAisne;

3) Secteur de vallée touristique entre Vic et l'entrée de Soissons;

4) Secteur de vallée urbanisée entre l'entrée et la sortie de Soissons;

\section{$7 /$}

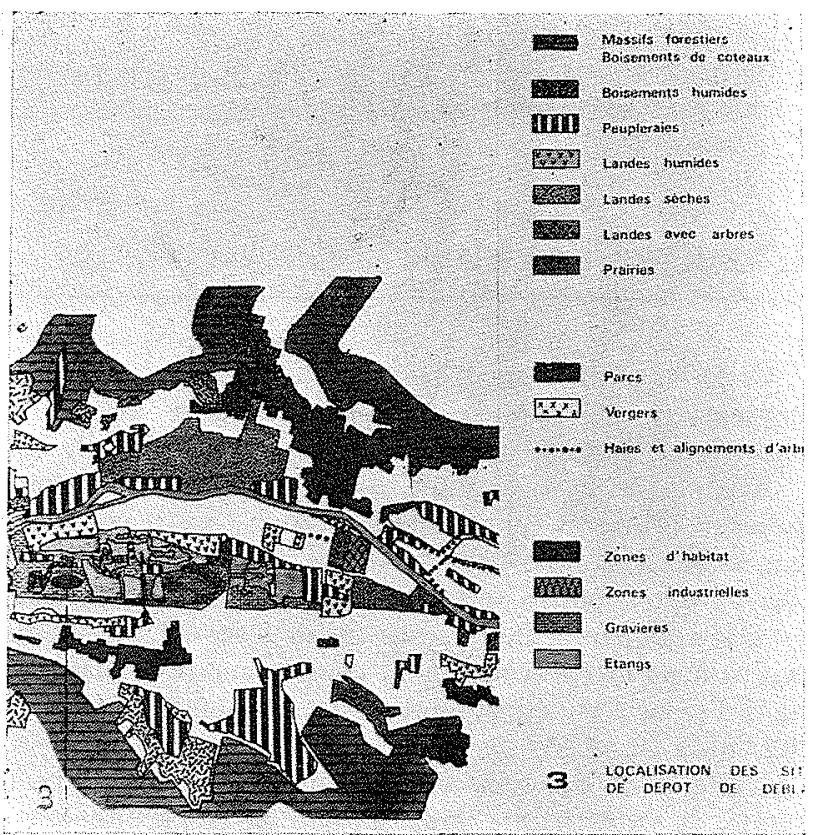

5) Secteur de vallée naturelle, depuis Soissons jusqu'à Berry-au-Bac;

6) Enfin un nouveau secteur de vallée industrielle entre Berry-au-Bac et Reims.

\section{Etude particulière de l'ensemble forestier Compiègne, Laigue, Ourscamps}

En relation avec la prise en compte de l'intérêt des milieux naturels et des paysages, l'étude de certains secteurs de vallée a été approfondie.

En ce qui concerne l'ensemble forestier de CompiègneLaigue-Ourscamps, l'étude des formations végétales a montré l'extrême diversité des associations résultant de la diversité du substrat. Ces associations ont atteint, dans la plupart des cas, leur stade d'équilibre et se répartissent en trois séries de végétation principales:

- la chênaie-ormaie occupant le fond alluvial;

- la chênaie-charmaie occupant les zones d'altitudes comprises entre 25 et $70 \mathrm{~m}$;

- la hêtraic occupant les zones d'altitudes supérieures à $70 \mathrm{~m}$.

L'étude démontre également l'importance de l'ensemble forestier constitué par les deux massifs au point de vue cynégétique. De François I ${ }^{\text {er }}$ à Louis XVI, plus de $1200 \mathrm{~km}$ de chemins sablonnés, utilisés pour la chasse à courre, ont été tracés.

De nos jours ce sport continue d'être pratiqué. Sa tradition est maintenue par de brillants équipages.

La reconstitution des parcours a permis de mettre en évidence la zone de traversée rituelle de l'Aisne utilisée par les animaux (cerfs, sangliers) pour passer d'un massif à un autre (fig. 9).

L'étude a, par suite, proposé la protection de cette zone de passage, l'aménagement des berges de telle manière que les animaux puissent continuer de passer d'une rive à l'autre. Cet aménagement sera le plus simple, le plus naturel (et le moins coûteux...) possible puisqu'il s'agira de berges en terre à très faible pente, engazonnées.

Enfin, l'étude a mis en relief les zones sensibles composées d'interstices ou de périphéries agricoles dont la protection serait nécessaire pour maintenir la continuité écologique de l'ensemble forestier.

\section{Etude particulière des méandres de l'Aisne}

Un autre problème d'importance fondamentale est posé par la conservation du cadre naturel représenté par les méandres de l'Aisne, entre Bourg et Comin et Berry-au-Bac, cadre qui permet la pratique de nombreuses activités, telle que la pêche, le camping, la baignade le long des nombreuses plages de galets ou de sable qui parsèment les coudes de l'Aisne.

La diversité et la complexité des paysages attenant aux méandres (fig. 10) auraient été menacées de disparaître par 

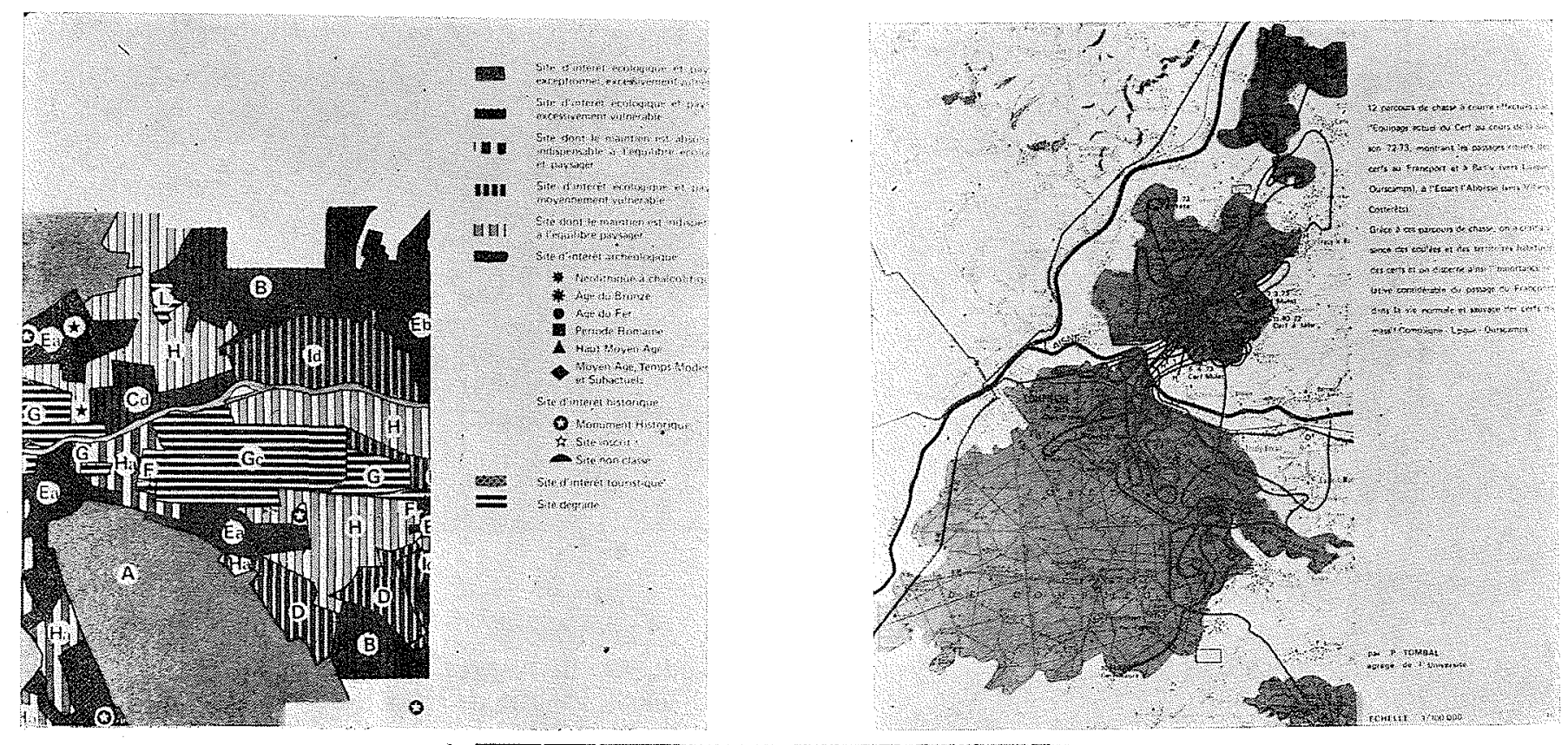

$8 /$

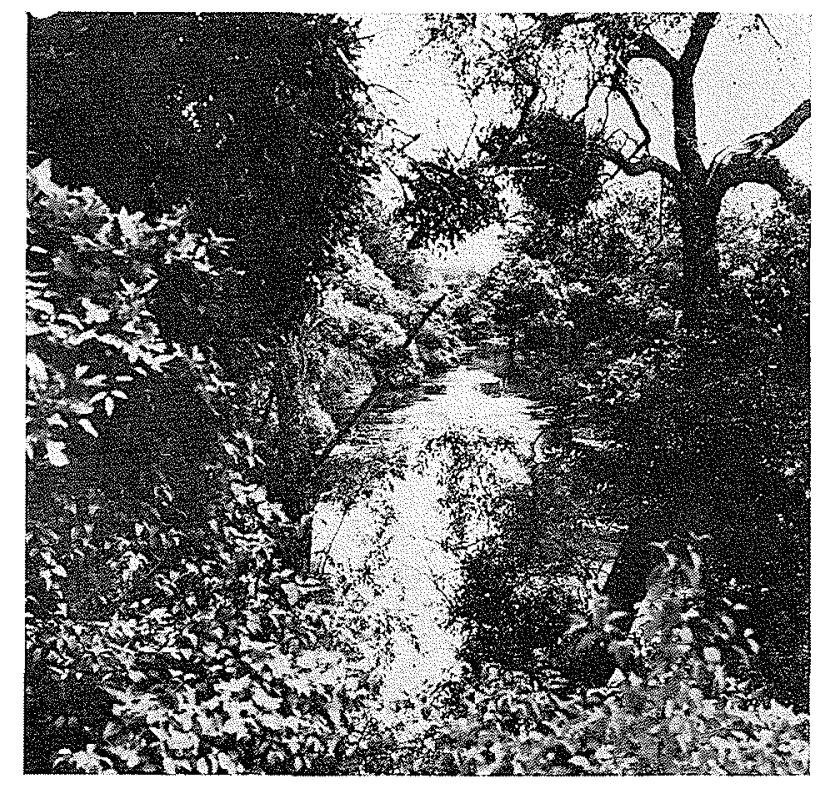

9/

11/

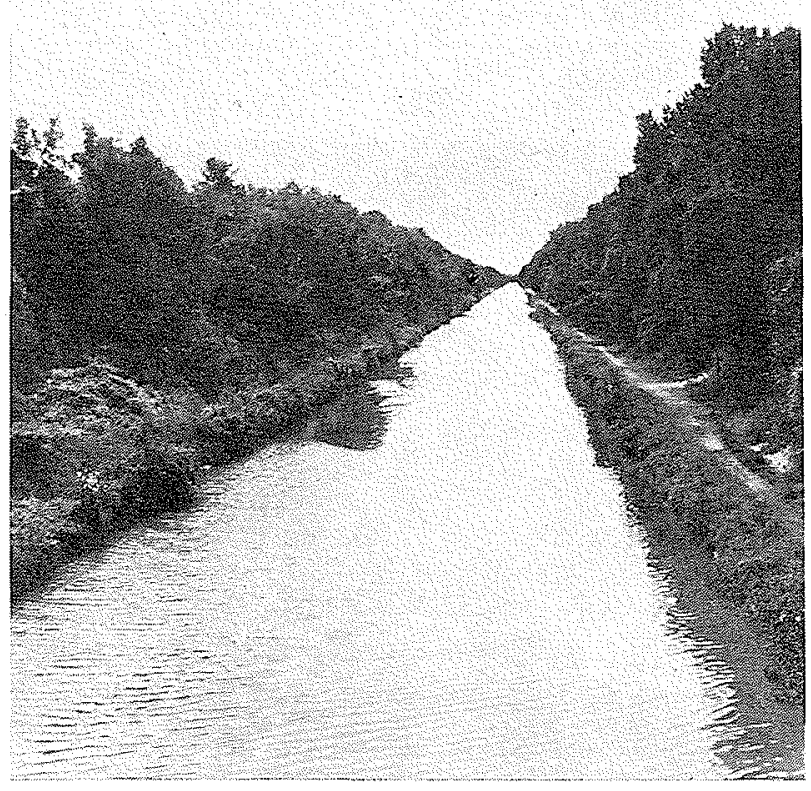

$10 /$

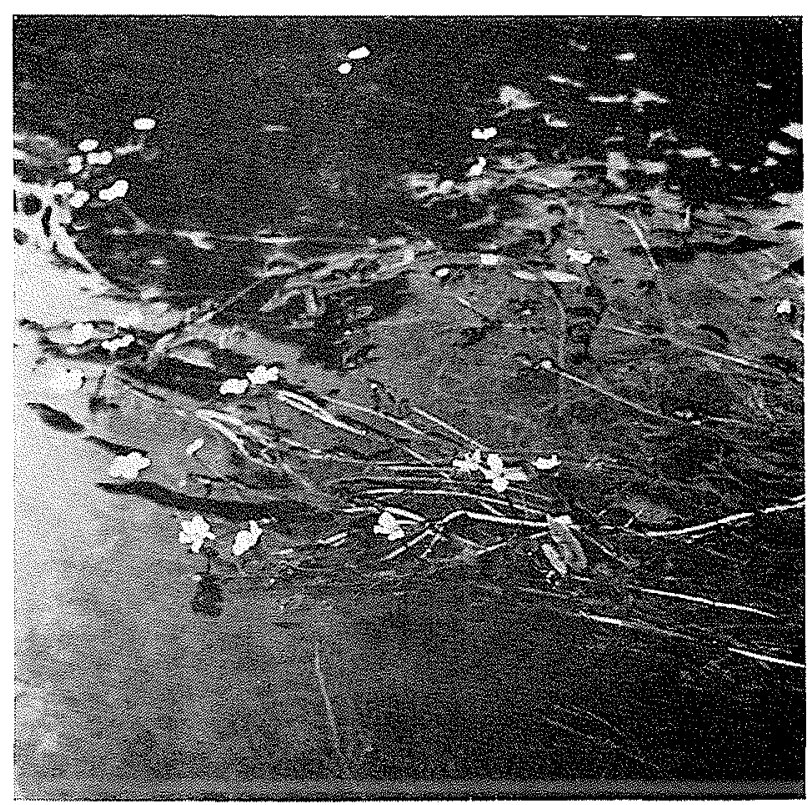




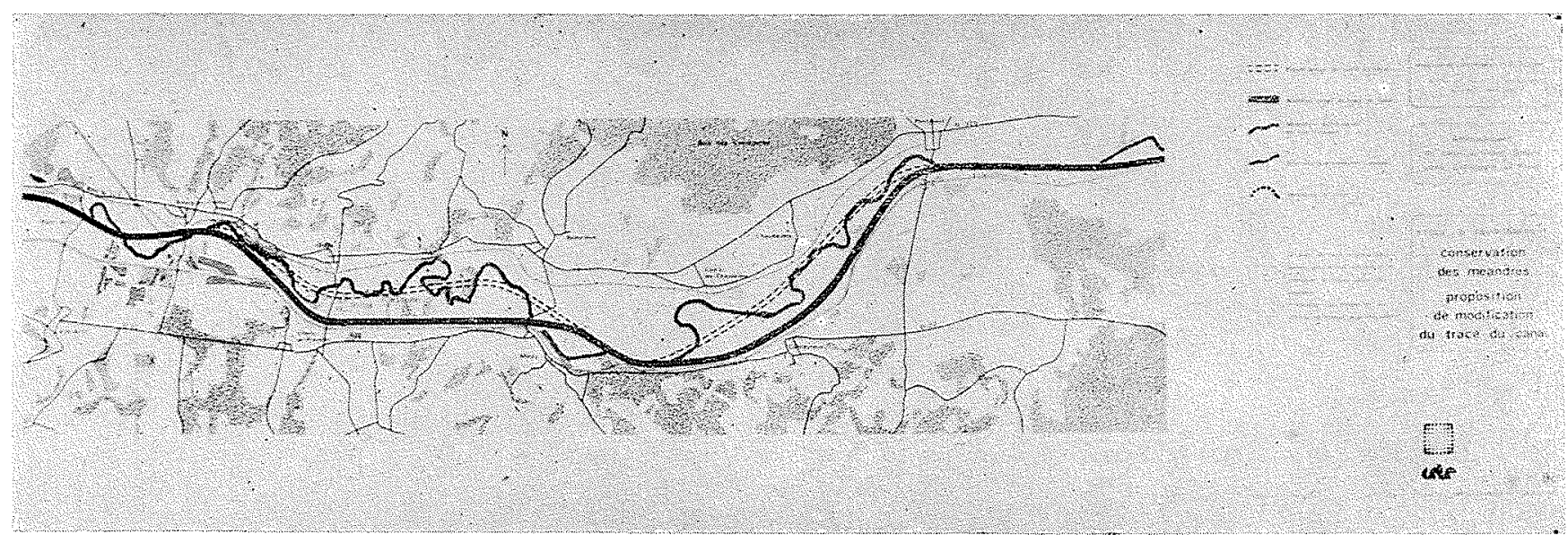

$13 /$

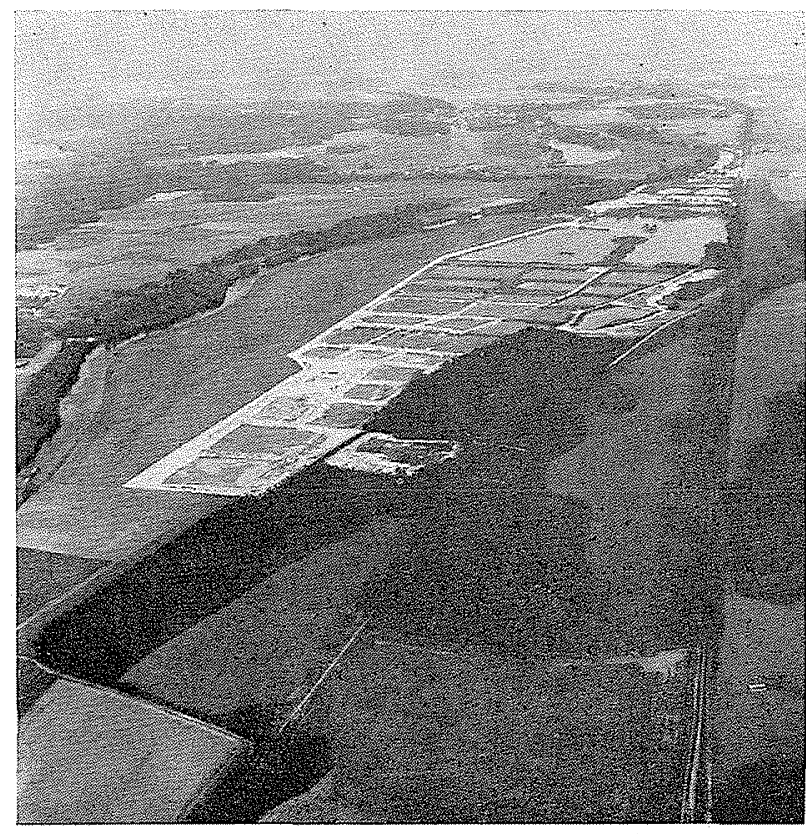

$14 /$

$15 /$

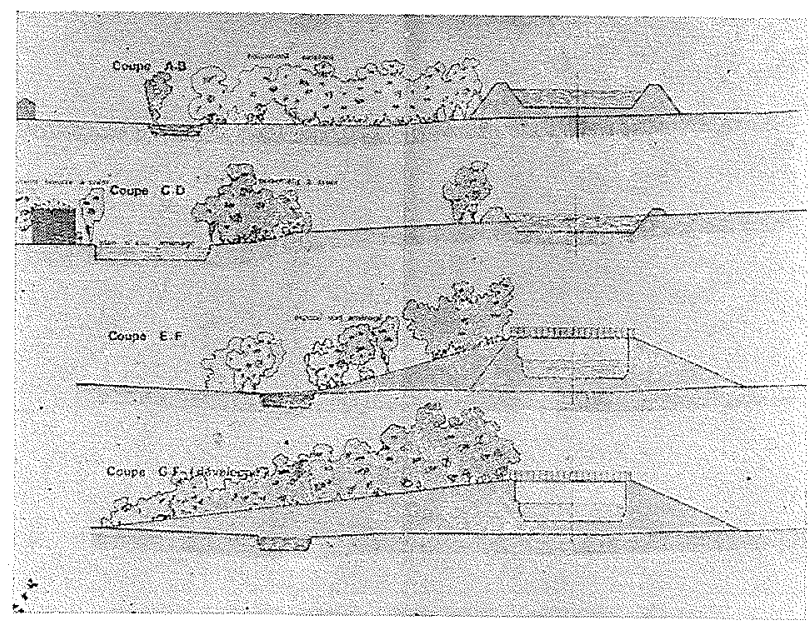

l'introduction de l'espace rectiligne du futur canal, dont le tracé initial scindait de nombreuses courbes de la rivière actuelles. Il suffit pour le comprendre de comparer l'Aisne rivière à l'actuel canal latéral (fig. 11).

La végétation naturelle qui borde l'Aisne, pleinement développée et adaptée aux conditions du milieu, contribue à la fixation des berges: le peuplier américain rejette vigoureusement de souche, dressant ses nombreux troncs sur une longueur de rive de plusieurs mètres; dans la même zone de battement des eaux correspondant aux périodes de crues et d'étiage, on trou've le saule des vanniers, tandis que sur le haut des berges se développe le saule blanc mêlé au saule fragile.

Dans l'eau pure de la rivière, de nombreuses plantes aquatiques se pressent, telles la renoncule d'eau, ici en pleine floraison (fig. 12).

L'aménagement des méandres aurait entraîné la disparition des milieux naturels et des paysages qui en dépendent, et dans tous les cas cet aménagement se serait révélé peu satisfaisant.

Aussi une modification du tracé évitant le scindement des méandres a-t-elle été élaborée (fig. 13). La présence de la future écluse de Pargnan en facilite l'application; en effet la rivière actuelle pourra être alimentée gravitairement par

\section{$16 /$}

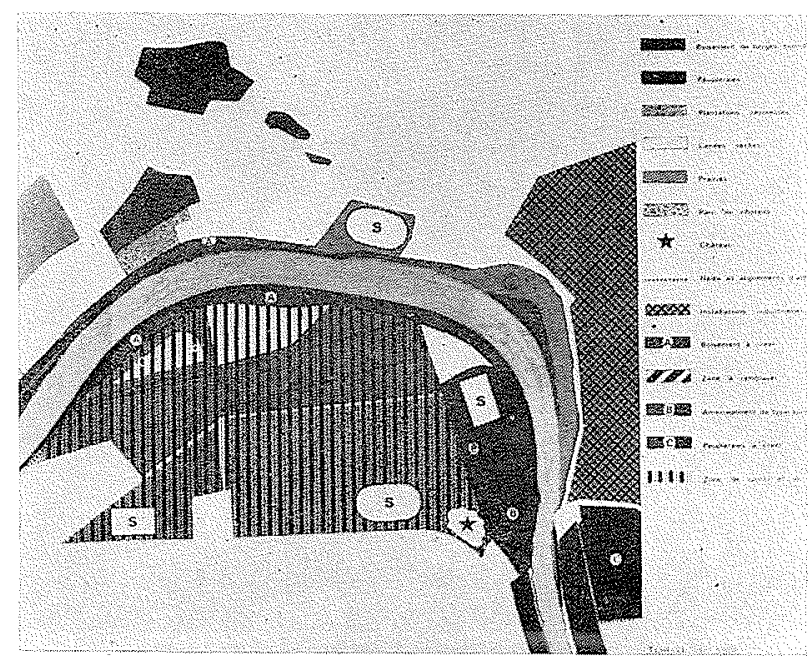


le bief amont et s'écoulera gravitairement également dans le bief aval.

Une étude complémentaire a également été entreprise, avec l'aide des ingénieurs de la première région piscicole, afin de déterminer les zones sensibles pour la reproduction et le maintien de la faune aquatique. Le nouveau projet adopté permet d'éviter toutes les zones fréquentées par les pêcheurs et épargne toutes les zones de reproduction, sauf une frayère à brochets qu'il sera sans doute possible de rétablir artificiellement.

\section{Le problème des déblais}

Les déblais provenant de la construction de la nouvelle voie d'eau seront utilisés pour le modelage des berges, en harmonie avec le terrain naturel, ou pour créer des volumes s'intégrant parfaitement aux mouvements du terrain naturel.

Ce problème des déblais, dont l'excédent est de l'ordre de 25 millions de $\mathrm{m}^{3}$, se pose sur toute la longueur du tracé.

Un recensement exhaustif des gravières pouvant être utilisées comme zones de dépôts a été effectué (fig. 14).

Le choix de ces gravières a été effectué en tenant compte de leur capacité et de leur proximité par rapport au canal et on s'est efforcé, dans toute la mesure du possible, de ne pas toucher aux gravières aménagées en zones de loisir ou pour la pêche.

Certaines gravières, une fois remblayées, seront rendues à l'agriculture ou pourront être aménagées en zones nouvelles de loisirs.

\section{Quelques amémagements particuliers}

Suivant le tracé proprement dit de la voie à grand gabarit, des aménagements ponctuels sont également prévus qui tiennent compte des potentialités du site. On peut citer par exemple :
- la création d'un espace vert sur l'emplacement d'une zone résiduelle située à Loivre, entre le canal actuel de l'Aisne à la Marne et le futur canal situé en fort remblai : les pentes seront adoucies, les terrains modelés et plantés (fig. 15);

- la reconstitution de la végétation des rives grâce à l'étude détaillée de la végétation existante et potentielle qui a été réalisée;

- l'aménagement aux points de traversée, par exemple pour le pont de Choisy-au-Bac, avec préservation des vues perspectives sur la forêt de Laigue;

- l'aménagement de la pénétration dans Soissons, appuyé sur l'espace prévu au schéma d'aménagement et d'urbanisme, à l'emplacement de l'ancien aérodrome, et sur les équipements sportifs existants, et qui consiste en outre à reconstituer et à élargir les Ianières boisées le long de chaque rive, à conserver, à agrandir et à planter l'île située en face de la zone industrielle, de manière à créer un couloir de verdure pénétrant jusqu'au cœur de l'agglomération (fig. 16).

$$
\text { : }
$$

L'étude qui a été réalisée par le Centre d'Etudes Techniques de Lille, sous la direction du Service de Navigation de Compiègne, a permis de confirmer et de comprendre l'importance de sites déjà connus, comme le massif forestier de Compiègne-Laigue-Ourscamps.

Elle a aussi permis de découvrir des sites exceptionnels inconnus du grand public — et des ingénieurs responsables du projet... -, excessivement vulnérables, comme les méandres de l'Aisne. Grâce à la collaboration qui s'est établie entre les ingénieurs, les écologistes, les pêcheurs et leurs représentants et quelques associations (à l'enthousiasme parfois débordant), des résultats tangibles ont été obtenus.

Le compromis ainsi réalisé vaut d'ailleurs pour tout le projet; seules une étroite collaboration entre spécialistes et une concertation approfondie avec les populations riveraines pouvait permettre l'heureuse insertion d'un projet à vocation industrielle dans une nature à l'aspect encore presque sauvage par endroits.

\section{Discussion}

Président : M. J. CHAPON

Nous avons entendu, dit $M$. le Président, une communication particulièrement intéressante, et j'en profite pour féliciter le Service de la Navigation de Compiègne pour l'originalité de cette étude et le sérieux avec lequel elle a été menée. C'est un exemple extrêmement probant de la volonté de ne pas faire n'importe quoi, même si cela doit coûter plus cher. Il n'est d'ailleurs pas évident que cela va coûter plus cher...

Messieurs, je vous laisse la parole pour les auestions aue vous désirez poser.

M. JAMME (Inspecteur Général de l'Environnement) intervient en ces termes:

Depuis plusicurs années déjà, une étude denvironnement est imposée aux U.S.A. lorsqu'un projet a une incidence notable sur l'environnement.

Les organismes financiers internationaux imposent également cette étude lorsqu'ils participent au financement de ce genre d'ouvrages. Jai en personnellement ì traiter de tels problèmes s'agissant de projets de construction de barrages au Cameroun et en Ethiopie, projets étudiés par EDF.

En France, on commence à s'intéresser à ces questions. Le rapport de la Commission Gruson, sur le gaspillage, propose d'imposer de telles études lorsque l'Etat participe au financement. Je me suis insurgé contre cette dernière restriction; le mode de financement n'a rien à voir à l'affaire.

Le Service de la Navigation de Compiègne a été un précurseur 
en la matière. Je voudrais donc joindre mes félicitations à celles que M. le Président lui a adressées tout à l'heure.

M. Arignon (Conseil Supérjeur de la Pêche, Compiègne) félicite M. Cocozza et le CETE de Lille d'avoir examiné si attentivement les problèmes paysagers et écologiques posés par leurs importants travaux.

Cependant, dit-il, le canal est un vecteur, mais également un milieu aquatique vivant; dans le cas du choix des méandres conservés pour la beauté du paysage et pour l'accueil des pêcheurs à la ligne, des critères biologiques ont-ils été retenus et étudiés par le CETE, auteur de l'étude?

Une étude hydrobiologique piscicole et halieutique, aussi fine que l'étude paysagère, permettrait de mettre en harmonie les impératifs hydrauliques et les impératifs hydrobiologiques et halieutiques. Cette mise en harmonie pourrait limiter les réactions d'usagers - les pêcheurs - dont le domaine de prospection est déià très limité.

II s'agit donc du souhait de voir les études hydrobiologiques et piscicoles prises en compte lors de l'étude générale de tels projets d'aménagement de cours d'eau.

Je dois dire, répond $M$. CocozzA, que nous nous sommes surtout attachés à la conservation du paysage aui est vraiment d'une grande beauté. Mais nous avons discuté de la conservation du poisson avec des Présidents de Fédération de pêche - avec M. Mailly, notamment - qui nous a proposé d'heureuses modifications au projet initial. C'est ainsi que nous avions envisagé - afin d'augmenter la hauteur d'eau - de draguer certains bras de la rivière, ce qui aurait conduit nous dit-il à «massacrer le poisson ». On a donc laissé les fonds en l'état... ce qui a permis certaines économies.

Si l'avancement du projet, dit M. Arrignon, n'en est pas encore au point qu'on ne puisse pas examiner la question sur un plan purement hydrobiologique, je pense que ce serait certainement une voie à des solutions satisfaisantes pour tous.

Notre Service est tout prêt à poursuivre les discussions dans cette voie, conclut $\mathrm{M}$. CocozzA.

M. P. Chevrier (Directeur honoraire EDF et Président de la SHF) rappelle, en ces termes, une expérience vécue il y a 25 ans, concernant la nécessité de ménager aux gros mammifères sauvages la possibilité de traverser un canal:

Le canal d'amenée d'eau de la centrale hydroélectrique de Witaker - sur un affuent de la Meuse - long de 6 à $7 \mathrm{kll}$, comportait des berges revêtues en béton, inclinées à $45^{\circ}$, empêchant toute remontée d'un animal tombé à l'eau. Résultat: une centaine d'animaux sauvages noyés par an.

On y remedia en installant des ponts improvisés, faits de baliveaux recouverts de terre et de mottes d'herbe pour permettre le franchissement, et des escaliers de sauvetage de place en place avec des perches flottantes pour guider l'animal en difficulté vers lescalier.

Je félicite les bâtisseurs d'aujourd'bui de prévoir, dans leurs projets, la solution de ces problèmes, dont malheureusement on ne se sonciait pas à l'avance, autrefois.

Nous avons déjà eu des contacts, dit M. Cocozza, avec les Associations de chasseurs des régions où des problèmes de ce genre peuvent se poser; d'un commun accord, il a été décidé de ne procéder à des aménagements que lorsque les endroits de passage privilégiés des animaux auront été détectés; facile en forêt de Compiègne, grâce aux comptes rendus de chasses à courre, cette détection est beaucoup plus délicate sur des dizaines de kilomètres du tracé. D’ailleurs, si l'industrialisation prévue se réalise, il est peu probable que les gros animaux continuent à passer à certains endroits.

Nous sommes conscients du problème et avons présenté et étudié. avec le Conseil Supérieur de la Chasse et diverses Associations locales, plusieurs solutions qui ont déjà fait leur preuve dans l'Est de la France.

Se référant aux travaux à poursuivre dans les prochaines années pour l'étude de la liaison Rhin-Rhône (notamment dans les vallées de l'Hl et du Doubs), M. Marchal souligne l'intérêt d'une comparaison approfondie de deux méthodes d'investigation sur l'environnement qui ont été évoquées au cours de la présente Session:

- celle poursuivie par une équipe pluridisciplinaire comme celle du CETE de Lille;

- celle confiée à un Centre universitaire, tel que celui de Besançon dont nous a parlé, hier, M. VerneauX.

M. le Président clôt la discussion sur les remarques ci-après:

La protection de la nature c'est, en particulier, celle de la faune, c'est un peu une langue d'Esope, c'est le meilleur et c'est le pire, et on peut en tirer ce que l'on veut comme ce que l'on ne veut pas.

Le Service de la navigation de Compiègne s'est attiré les compliments parce au'il a établi un projet avec la préoccuation d'éviter que les bêtes poursuivies par une chasse à courre risquent de se noyer, en ne pouvant pas remcnter sur la berge de la voie d'eau qu'elles traversent.

Croyez-vous qu'on ait vraiment fait cuvre utile et que la noyade soit une fin plus tragique que celle que connaissent ces animaux, forcés par une vingtaine de chiens hurlants et une douzaine de cavaliers, jusqu'à ce que le souffle leur manque, pour finalement être servies d'un coup de dague? Et dans le degré de «cruautés relatives », on peut trouver des exemples analogues dans le domaine de la pêche.

Tout cela montre combien est subjective la notion de protection de la nature, on peut dire la même chose des questions d'environnement. Parlons-en avec modestie, chaque fois que nous jugeons une création humaine: jamais la main de l'homme ne sera aussi habile que celle du Créateur.

Ce que doit faire l'homme, c'est précisément mettre son intelligence all service de la nature, en essayant de sauvegarder ce que lui a donné le Créateur mais sans oublier qu'il doit agir pour le bien des autres hommes. D'aucuns regretteront toujours les interventions humaines: une émission de télévision a récemment repris le livre d'un auteur qui regrette les transformations résultant de l'aménagement du Rhône dont les rives ne sont évidemment plus ce qu'elles étaient. L'auteur qui critique le travail des hommes refuse-t-il l'électricité et continue-t-il à s'éclairer à la bougie ou à la torche?

Je conclue mon propos en rappelant notre double devoir do protéger le capital inestimable qui nous a été légué - et ici, je dirai plus particulièrement nos rivières - et de travailler pour le bonheur des hommes en faisant abstraction de nos intérêts individuels. il faut que nos agriculteurs admettent de céder un peu de leurs terres pour permettre de construire une nouvelle voie d'eau en préservant le caractère d'une rivière naturelle; il faut que les amoureux de la nature admettent qu'en certains endroits les nouvelles voies d'eau empruntent les rivières pour éviter de trop mordre sur des terrains agricoles de grande valeur.

J'observe simplement que, dans le cas de la voie Compiègne-Reims, cette double préoccupation rapproche le tracé de celui de lancien canal... Peut-être nos anciens, qui ne connaissaient pas encore les termes «d'environnement ou d'écologie», en faisaient-ils comme M. Jourdain, sans le savoir ? Peut-être obtenaient-ils ce résultat heureux parce qu'ils travailiaient plus lentement, de façon olus sereine et finalement avec plus de sérieux? Cela devrait nous servir do leçon!

Le Président donne ensuite la parole à M. Clément pour l'exposé de sa communication. 